\title{
Extraction of Vegetation Structure and Surface Parameters using Polarimetric and Interferometric SAR Techniques
}

\author{
L. Sagués* , X. Fàbregas* , A. Broquetas* \\ * Dpt. Teoria del Senyal i Comunicacions, Universitat Politècnica de Catalunya (UPC) \\ Campus Nord UPC, D3 Building, c/ Jordi Girona, 1-3, 08034 Barcelona, Spain \\ Phone / Fax: 34934011065 / 7232, E-mail: sagues@tsc.upc.es \\ ** Institute of Geomatics \\ Parc de Montjuïc, E-08038 Barcelona, Spain, E-mail: toni@tsc.upc.es
}

\begin{abstract}
ABSRACT
The paper presents different polarimetric and interferometric inversion radar approaches to extract the characteristics of vegetation and bare soil surfaces. The inversion techniques here presented make use of multifrequency, radar interferometric and polarimetric data to increase the number of input data. The proposed techniques have been experimentally tested under laboratory conditions as well as in real outdoor scenarios.
\end{abstract}

\section{INTRODUCTION}

An important application of radar polarimetry is the retrieval of physical parameters related to natural scattering mechanisms of the surface under observation. There are several inversion techniques that have been to extract the surface roughness and volumetric moisture of bare soil surfaces from polarimetric data [1], [2]. However, such algorithms are semi-empirical models based on the firstorder scattering approximation (pure surface scattering), ignoring the effect of multiple scattering. Therefore, these algorithms should provide more accurate results after separating the pure surface scattering mechanism from those processes associated with multiple scattering effects by means of a decomposition technique [3].

In case of random media, like vegetation, the electromagnetic waves interaction with scatterers is a more complex process that is sensitive to a much larger number of target parameters. Consequently, the estimation of vegetation parameters can not be achieved by using fixed polarization single frequency SAR data, even when a simple homogeneous one-layer model is considered. In this way, some polarimetric, multibaseline and multifrequency inversion algorithms have been proposed in order to extend the number of independent parameters [4], [5], [6].

In this paper we show a comparison of these inversion techniques from an experimental point of view, using indoor and outdoor experimental results. Some multifrequency experiments were carried out in the anechoic chamber of the European Microwave Signature Laboratory (EMSL) at JRC (Ispra, Italy), where samples of maize were measured at L, S and $\mathrm{C}$ bands. Furthermore, other outdoor measurements were carried out with a ground-based fully polarimetric scatterometer developed at the UPC (Barcelona, Spain) [7]. The measurements were conducted on bare rough surfaces and sorghum fields.

\section{BARE SURFACES}

There are several approaches in the literature which make use of semi-empirical models to extract surface roughness and moisture from polarimetric SAR data. In this paper we will present the results obtained by applying the method proposed by Dubois et al in [2]. This model only uses the copolarized backscattering coefficients and thus, it will be only valid under pure surfaces scattering conditions characterized by low entropy [8]. However, it is possible to find surface scattering processes with higher entropy due to changes in the surface roughness, as occurs when the roughness or correlation length of the surface increases. Moreover, surface scattering after propagation through an anisotropic random media, like vegetation will also generate a high entropy process. In all of these cases the model proposed in [2] leads to incorrect results because the surface under observation is characterized by multiple scattering effects that generate an adequate cross-polarized component.

To solve this problem, we can isolate the pure surface scattering mechanism from the volumetric effects by means of a target decomposition before applying the inversion method [3]. This can be performed by decomposing the original covariance matrix that describes the natural surface as a sum of three orthogonal covariance matrices:

$$
[C]=\lambda_{1} u_{1}^{+}+\lambda_{2} u_{2}^{+}+\lambda_{3} u_{3}^{+}=[C]_{1}+[C]_{2}+[C]_{3}
$$

where $\lambda_{1}>\lambda_{2}>\lambda_{3}$ are the real eigenvalues and $u_{1}, u_{2}, u_{3}$ the corresponding eigenvectors. Thus, the scattering process that describes the original natural surface has been decomposed as a sum of three independent scattering mechanisms. The dominant scattering mechanism (first eigenvalue) will be associated with a pure surface scattering mechanism whereas the rest correspond to multiple scattering processes. 
In order to test this inversion model, two bare soils were measured at Castellterçol (Barcelona) in October 1999. During the measurement campaign, accurate ground truth data were collected over these test fields. The size of the three test fields was $10 \times 10 \mathrm{~m}$ and the distance between the antennas and the ground was 18 meters, covering an incident angle range from 50 up to 70 degrees. Fully polarimetric data were collected at $\mathrm{C}$ and $\mathrm{X}$ bands with a ground-based scatterometer that can operate as a Circular SAR system [7]. Fig. 2 shows the $\mathrm{H} / \alpha$ decomposition [8] corresponding to the measured original surface and those three scattering processes derived from the decomposition described in (1). As can be seen, the measured surface is characterized by a high entropy process. However, the three scattering process derived from the decomposition are located at different zones inside the $H / \alpha$ plane. The dominant scattering mechanism is found at zone 9 , which corresponds to pure surface scattering, whereas the rest appear at other zones that are characterized by multiple scattering effects. As can be derived from the results shown in Table I and Table II, the application of this previous target decomposition process makes the surface parameter estimation more precise.

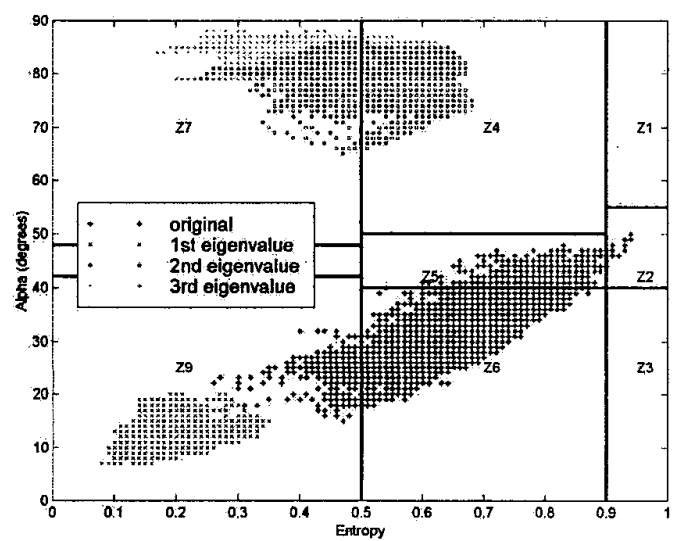

Figure 1: $\mathrm{H} / \alpha$ decomposition corresponding to a natural surface

\begin{tabular}{|c|c|c|c|c|}
\hline \multicolumn{3}{|c|}{ Table I : Results before decomposition (C-band) } \\
\hline \multirow{2}{*}{$\begin{array}{c}\text { Test } \\
\text { field }\end{array}$} & \multicolumn{2}{|c|}{ RMS height (cm) } & \multicolumn{2}{c|}{ Volumetric moisture } \\
\cline { 2 - 5 } & Measured & Estimated & Measured & Estimated \\
\hline Bare soil 1 & 1.0215 & 8.66 & 0.2178 & 0.3416 \\
\hline Bare soil 2 & 1.4712 & 11.56 & 0.2060 & 0.3144 \\
\hline
\end{tabular}

Table II : Results after decomposition (C-band)

\begin{tabular}{|c|c|c|c|c|}
\hline \multirow{2}{*}{$\begin{array}{c}\text { Test } \\
\text { field }\end{array}$} & \multicolumn{2}{|c|}{ RMS height (cm) } & \multicolumn{2}{c|}{ Volumetric moisture } \\
\cline { 2 - 5 } & Measured & Estimated & Measured & Estimated \\
\hline Bare soil 1 & 1.0215 & 1.09 & 0.2178 & 0.2805 \\
\hline Bare soil 2 & 1.4712 & 1.94 & 0.2060 & 0.2465 \\
\hline
\end{tabular}

\section{VEGETATION}

If we model a surface covered by vegetation as a layer of randomly oriented particles over the ground, the backscattered field from a cluster along the $\mathrm{z}$-axis is composed of four components as depicted in Fig. 2. Once the total backscattered field has been calculated in the frequency domain, we can calculate the complex Frequency Correlation Function (FCF) [6] by correlating independent sample measurements at different frequency points (multifrequency method):

$$
E\left\{E_{p p}\left(f_{1}\right) E_{p p}^{*}\left(f_{2}\right)\right\}=e^{-2 \kappa \frac{h}{\cos \theta}}\left[\begin{array}{l}
\frac{e^{\chi h}-1}{\chi}\left(1+\left|R_{p}\right|^{4} e^{-\chi h}\right)+ \\
\left(4\left|R_{p}\right|^{2} Q_{p}+M_{p}\right) h
\end{array}\right]
$$

where $h$ is the height of the vegetation volume, $\kappa$ the mean extinction coefficient $R_{p}$ the coherent reflectivity of the ground surface, $M_{p}$ the ground-to-volume amplitude ratio and $Q_{p}$ the ratio between the bistatic and backscatterer phase matrix copolarized elements [6] and

$$
\chi=2(\kappa \cos \theta+j(4 \pi / c) \Delta f \cos \theta)
$$

being $\Delta f=f_{2}-f_{1}$. The FCF function described in (2) is formed by four terms which are related to different scattering processes. The first one is associated with the direct backscattering from the volume particles, the second one is the ground-volume-ground term, the third one corresponds to the bistatic scattering contribution due to the double bounce between the volume particles and the ground, whereas the last term is the direct backscattering from the ground.

We can also express the backscattered field in the timespace domain and calculate the interferometric coherence by correlating two SAR images acquired from different viewing angles $\theta_{1}$ and $\theta_{2}$. As a result of this, the derived complex correlation function has the same expression as depicted in (3) with the inclusion of the conventional. interferometric phase term $\phi_{\text {int }}$ and considering that:

$$
\chi=2(\kappa \cos \theta \cos (\Delta \theta / 2)-j(2 \pi f / c)(\Delta \theta / \sin \theta))
$$

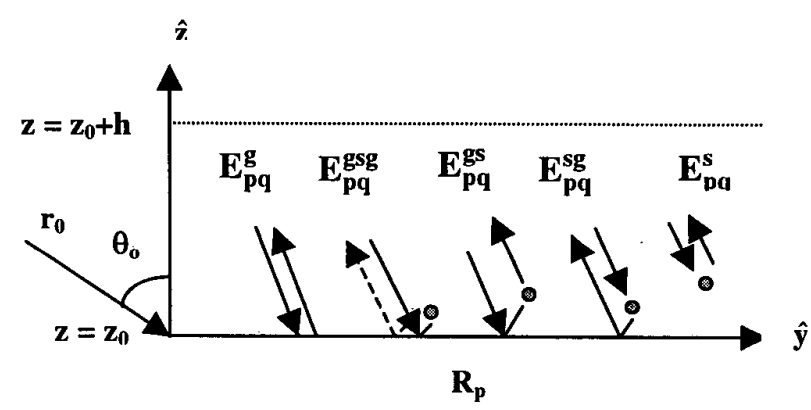

Figure 2: Backscattered electric fields in a random medium 
In this case the number of interferometric observables can be increased by measuring from other viewing angles (multibaseline method) [4] or by collecting fully polarimetric data (POLINSAR method). However, since some of these parameters depend on the polarization, we can not use the complete scattering model described in (2). Indeed, if we collect single baseline fully polarimetric data, we must consider a simpler model presented in [5] which only takes into account the first and the last terms of (2), i.e. neglecting the volume-ground interaction effects.

These inversion techniques were experimentally tested under laboratory and outdoor conditions by applying both FCF (see Table III, IV and V) and POLINSAR (see Fig. 3 and Table VI) methods. As can be derived from the indoor results, both approaches lead to good results. However, the parameter estimation is more accurate if lower frequency bands are used and the complete scattering model is considered. Otherwise, the POLINSAR inversion algorithm fail when applied to outdoor measurements due to temporal decorrelation effects caused by the time interval between two acquisitions and the oscillation movement of the crops.

Table III : Indoor Results using the FCF (S-band)

\begin{tabular}{|c|c|l|c|}
\hline Maize $\mathrm{h}=1.2 \mathrm{~m}$ & \multicolumn{1}{|c|}{$h(\mathrm{~m})$} & \multicolumn{1}{c|}{$\kappa\left(\mathrm{m}^{-1}\right)$} & \multicolumn{1}{c|}{$M$} \\
\hline Complet model & 1.2202 & 0.9952 & 2.1920 \\
\hline Simple model & 1.5827 & 0.4542 & 0.3598 \\
\hline
\end{tabular}

Table IV : Indoor Results using the FCF (C-band)

\begin{tabular}{|c|l|l|c|}
\hline Maize $\mathrm{h}=1.2 \mathrm{~m}$ & \multicolumn{1}{|c|}{$h(\mathrm{~m})$} & \multicolumn{1}{|c|}{$\kappa\left(\mathrm{m}^{-1}\right)$} & $M$ \\
\hline Complete model & 1.4318 & 0.6856 & 0.5988 \\
\hline Simple model & 1.6120 & 0.4020 & 0.2537 \\
\hline
\end{tabular}

Table V : Outdoor Results using the FCF (X-band)

\begin{tabular}{|c|c|c|c|}
\hline Sorghum $\mathrm{h}=1.4 \mathrm{~m}$ & $h(\mathrm{~m})$ & $\kappa\left(\mathrm{m}^{-1}\right)$ & $M$ \\
\hline Complete model & 1.7964 & 1.1406 & 2.08 \\
\hline Simple model & 1.9587 & 1.0071 & 0.71 \\
\hline
\end{tabular}

Table VI : Outdoor Results using POLINSAR

\begin{tabular}{|l|c|c|c|}
\hline Sorghum & $h$ (Cloude) & $H$ (Pauli) & $h(\mathrm{H}, \mathrm{V})$ \\
\hline$h=1.4 \mathrm{~m}$ & 6.1266 & 9.9610 & 4.9470 \\
\hline
\end{tabular}

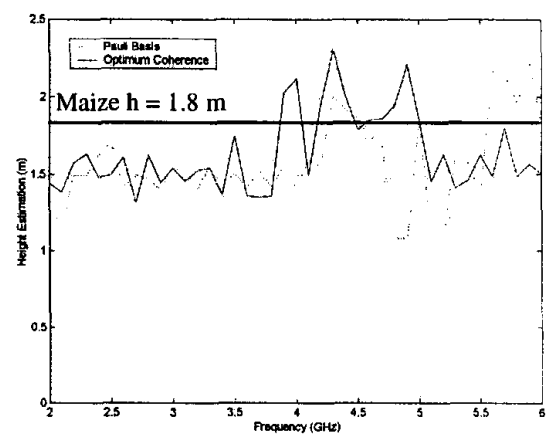

Figure 3: Height maize estimation by POLINSAR

\section{CONCLUSIONS}

Several inversion algorithms have been tested in this paper in order to extract some physical parameters of natural surfaces. First, we have shown that the performance of surface parameter inversion techniques can be improved by decomposing the measured surface into different elementary scattering mechanisms and selecting the pure surface one. Then, we have tested the application of two different approaches to extract the height of vegetation. The FCF method allows us to infer more vegetation parameters than the POLINSAR one but the knowledge of a precise DEM and an accurate system calibration is required. Otherwise, using the POLINSAR approach, we can retrieve the surface topography and some vegetation parameters. However, the vegetation height can be overestimated due to other coherence degradation effects.

\section{ACKNOWLEDGMENTS}

The authors wish to thank the CICYT (Spanish Commission for Science and Technology) ref TIC99-1050C03-01 for their financial support.

\section{REFERENCES}

[1] Y. Oh, K. Sarabandi, F. T. Ulaby, "An Empirical Model and an Inversion Technique for Radar Scattering from Bare Soil Surfaces", IEEE Transactions on Geoscience and Remote Sensing, vol. 30, no. 2, pp. 370-381, March 1992.

[2] P. C. Dubois, J. van Zyl, T. Engman, "Measure Soil Moisture with Imaging Radars", IEEE Transactions on Geoscience and Remote Sensing, vol. 33, no. 4, pp. 915-926, July 1995.

[3] I. Hajnsek, K. P. Papathanassiou, A. Reigber, S. R. Cloude, "Soil Moisture Estimation Using Polarimetric SAR Data", in Proc. IEEE IGARSS'99, Hamburg, 1999.

[4] R. N. Treuhaft, M Mohgaddam, J. J. Van Zyl, K. Sarabandi, "Vegetation Characteristics and Surface Topography from Interferometric Radar" Radio Science, vol. 31, pp. 1449-1485, 1996.

[5] P. Papathanassiou, A. Reigber, S. R. Cloude, "Vegetation and Ground Parameter Estimation using Polarimetric Interferometry", in Proc. of the ESA CEOS SAR Workshop Toulouse, 26-29 October 1999.

[6] K. Sarabandi, A. Nashashibi, "Analysis and Application of Backscattered Frequency Correlation Function", IEEE Transactions on Antennas and Propagation, vol. 37, no. 4, pp. 1895-1906, July 1999.

[7] L. Sagués, M. Bara, O. Mora, A. Broquetas, X. Fàbregas, "Circular Synthetic Aperture Radar (C-SAR) System and an Interferometric Algorithm for Ground-Based Applications", Proc. EUSAR'98, Friedrichshafen, Germany, pp. 499-502.

[8] S. R. Cloude, E. Potier, An Entropy Based Classification Scheme for Land Applications of Polarimetric SAR", IEEE Transactions on Geoscience and Remote Sensing, vol. 35, pp. 68-78, 1997. 